\title{
Antimicrobial Properties of Vertically Aligned Nano-Tubular Copper
}

\author{
Kafil Razeeb \\ University College Cork \\ Joanna Podporska-Carroll \\ Technological University Dublin \\ Mamun Jamal \\ University College Cork
}

See next page for additional authors

Follow this and additional works at: https://arrow.tudublin.ie/ehsiart

Part of the Biology Commons, and the Medicine and Health Sciences Commons

\section{Recommended Citation}

Razeeb, K. et al (2014). Antimicrobial properties of vertically aligned nano-tubular copper. Materials Letters, vol.128, pp.60-63. doi:10.1016/j.matlet.2014.04.130

This Article is brought to you for free and open access by the ESHI Publications at ARROW@TU Dublin. It has been accepted for inclusion in Articles by an authorized administrator of ARROW@TU Dublin. For more information, please contact arrow.admin@tudublin.ie, aisling.coyne@tudublin.ie,gerard.connolly@tudublin.ie.

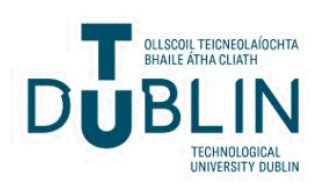




\section{Authors}

Kafil Razeeb, Joanna Podporska-Carroll, Mamun Jamal, Maksudul Hassan, Michael Nolan, Declan McCormack, Brid Quilty, Simon Newcombe, and Suresh Pillai 
Cite this article as Razeeb et al, Materials Letters, 128, 2014, 60-63

Full article is available at

http://www.sciencedirect.com/science/article/pii/S0167577X14007071

\title{
Antimicrobial Properties of Vertically Aligned Nano-tubular Copper
}

Kafil M. Razeeb, ${ }^{\text {a* }}$ Joanna Podporska-Carroll, ${ }^{\mathrm{b}}$ Mamun Jamal, ${ }^{\mathrm{a}}$ Maksudul Hassan, ${ }^{\mathrm{a}}$ Michael Nolan, ${ }^{\text {a }}$ Declan E. McCormack, ${ }^{\text {b,c }}$ Brid Quilty ${ }^{\mathrm{d}}$, Simon B. Newcomb ${ }^{\mathrm{e}}$ and Suresh C. Pillai ${ }^{\mathrm{b}, \mathrm{c}}$

${ }^{a}$ Tyndall National Institute, University College Cork, Dyke Parade, Maltings, Cork, Ireland

${ }^{b}$ Centre for Research in Engineering Surface Technology (CREST), FOCAS Institute, Dublin Institute of Technology, Kevin Street, Dublin 8, Ireland.

${ }^{c}$ School of Chemical and Pharmaceutical Sciences, Dublin Institute of Technology, Kevin Street, Dublin 8, Ireland.

${ }^{d}$ School of Biotechnology, Dublin City University, Glasnevin, Dublin 9, Ireland ${ }^{e}$ Glebe Scientific Limited, Newport, Co. Tipperary, Ireland

\begin{abstract}
In this work, the antimicrobial properties of vertically aligned nano-tubular $\mathrm{Cu}$ arrays (NT-Cu) fabricated via a template-based electrodeposition approach were investigated. The NT-Cu display good bactericidal activity against S.aureus and bacteriostatic properties against E.coli, S.sonnei, S.enterica and C.albicans. In contrast, $\mathrm{Cu}$-foil electrodeposited from the same solution
\end{abstract}

* Corresponding Author: kafil.mahmood@tyndall.ie 
shows low biological activity against the same microorganisms. The antimicrobial activity of NT-Cu depends on both the type of microorganism and exposure time. After 6 hours of exposure, over 99.99\% (log red $=4.43$ ) of S.aureus population was inactivated, whereas, for E.coli, S.sonnei, S.enterica and C.albicans it was 97.8, 94.2, 89.9, and 90.3\%, respectively.

Keywords: Nano-tubular copper, electrodeposition, bacteria, antimicrobial

\section{Introduction}

In recent years, there has been widespread interest into the effectiveness of nano-scale materials for antimicrobial applications, though there is still much work to be done [1-3]. It has been suggested that the bactericidal effectiveness of metal nanoparticles is due to both their size and high surface-to-volume ratio. Such characteristics should allow nanoparticles to interact closely with bacterial membranes, rather than being due solely to the release of metal ions [4]. Recently, gold nanoparticles and cuprous oxide micro-particles have been investigated for their antibacterial properties $[5,6]$. In the latter, $\mathrm{Cu}_{2} \mathrm{O}$ structures with different morphologies were studied and the effect of morphology on the antibacterial activity was reported [6].

Given the potential for nanostructured materials in antibacterial applications, this study investigates the antimicrobial properties of novel vertically aligned nano-tubular $\mathrm{Cu}$ arrays. Vertically aligned NT-Cu structure has high aspect ratio and ultrafine pores ( $\sim 25 \mathrm{~nm}$ ) compared to other structures of $\mathrm{Cu}$ (e.g. cu thin films and nanoparticles). Furthermore, the arrays are fabricated as film which does not require further processing to be used as antimicrobial surface. In this work, NT-Cu is fabricated using a template based electrochemical deposition method where the template is chemically removed after the fabrication of NT-Cu. The antimicrobial 
properties of these nano-tubular arrays were evaluated using selected pathogens: Gram-positive S.aureus, Gram-negative E.coli, S.sonnei, S.enterica and yeast C.albicans, showing antibacterial activity towards all pathogens with activity depending on pathogen and exposure time. Though interesting, the effect of array dimensions on antimicrobial properties is the subject of future work.

\section{Experimental}

The nano-tubular arrays were electrodeposited using anodic alumina oxide template with an average pore diameter of $\sim 300 \mathrm{~nm}$. The details of the fabrication and the elemental characterisation process are discussed in section 1 and 2 of the supplementary information. Evaluation of the antimicrobial properties of NT-Cu was performed using Gram-positive Staphylococcus aureus, Gram-negative Eschericshia coli, Shigellasonnei, Salmonella enterica and yeast Candida albicans. The procedure of assessments of the microbial properties is described in section 3 of the supplementary information.

\section{Results and Discussion}

Fig. 1(a) shows an SEM image of a blank nano-tubular array of $\mathrm{Cu}$ with an average height of $13 \pm 1 \mu \mathrm{m}$ and average diameter of $300 \mathrm{~nm}$ after removing the AAO template. The inset shows the TEM image of the ends of two nano-tubules seen in a sample in which the nano-tubules were dispersed onto a holey carbon support grid. Each tube exhibits characteristic V-shaped ends (indicated by “A”). The uniformity of the contrast seen across the width of the tube marked at B is consistent with the absence of a large hole in the Cu. Fig. 1(b) shows part of an ion milled sample cut at an oblique angle to the long axis of the nano-tubules. The general contrast 
uniformity in the fibre indicates the absence of a large hole running along the length of the NT.

However, a relatively small hole can be seen at B. Many of these structures exhibit the formation of holes with a typical diameter of $25 \mathrm{~nm}$ near their centres (but not exclusively so).
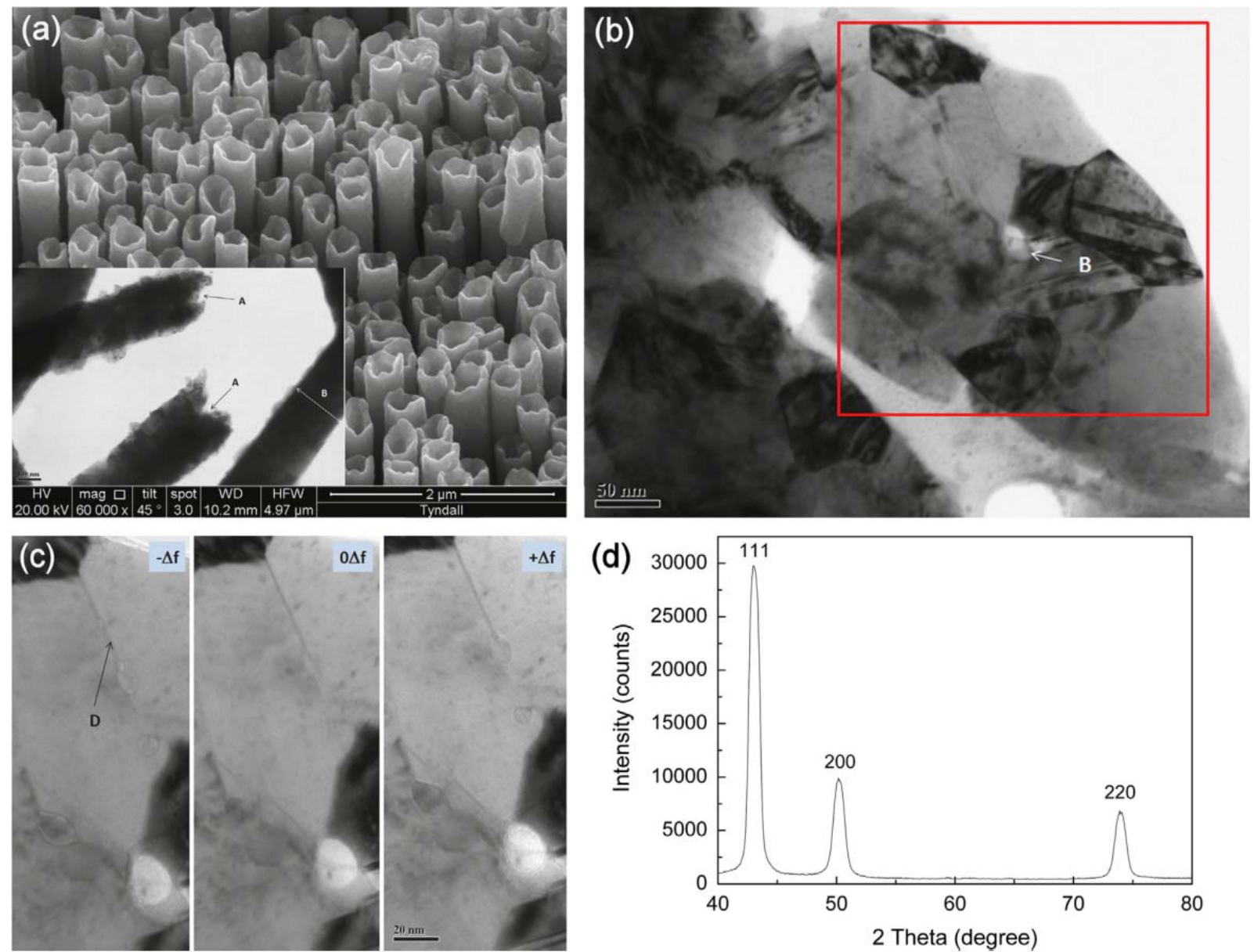

Fig 1. SEM images of (a) vertically aligned NT-Cu after removal of the template, inset TEM image of NT-Cu dispersed; (b) HRTEM image of single NT-Cu cut at an oblique angle to the long axis of the tube showing hole at B. (c) Defocus images taken from the boxed region in (b) showing Fresnel contrast from the grain boundary marked at D; (d) XRD pattern of the as deposited NT-Cu arrays.

Finer scale porosity is visible in all the samples. Fig. 1(c) shows the defocus images taken from the boxed region in 1(b) showing Fresnel contrast from the grain boundary marked at D. The 
boundary is either porous or decorated with a thin film of low scattering potential amorphous material. The NT-Cu has a generally fine grained microstructure ( $20-250 \mathrm{~nm})$ and no evidence was found that their formation takes place with a preferred crystallographic growth direction. The XRD analysis shows polycrystalline phases of (111), (200), and (220) orientations (Fig. 1(d)).

The antimicrobial evaluation was performed using S.aureus, E.coli, S.sonnei, S.enterica and C.albicans. The results of the preliminary evaluation on agar plates are shown in Fig. 2. Among the organisms used for the test, only S.aureus showed significant susceptibility to NT-Cu which was exhibited by a well-defined zone of inhibition of $15 \mathrm{~mm}$ observed around the test sample. For the other organisms examined, distinct zones of inhibition were not observed, however a reduction in the microbial population around the sample was clearly visible. This observation may indicate bacteriostatic (bacteria-inhibiting) rather than bactericidal (bacteria-killing) properties of the NT-Cu. When an electrodeposited Cu-foil was tested, no changes in agar surface appearance were observed for all microorganisms tested, indicating low biological activity of a $\mathrm{Cu}$ film.

To investigate obtained results in more detail, samples were removed from the agar after the experiment, and their surface was observed using Scanning Electron Microscopy. SEM analysis in Fig. 2(F-J) reveals that after 24 hours of experiment, the amount of S.aureus cells present was significantly lower in comparison with other organisms and in fact only individual, strongly damaged S.aureus cells were present on the NT-Cu. There was similar amount of the bioburden observed on other samples where areas with a significant microbial residue were observed. To determine the interaction between NT-Cu and the biological systems in suspension, a quantitative analysis was performed on the same type of materials exposed to microbial cultures 
suspended in a liquid medium. A summary of the quantitative evaluation of antimicrobial properties is presented in Table 1 and Fig. 3. The reduction of the microbial population is expressed as log value at each point of time. The lack of antimicrobial action is denoted as “zero”.

Table 1. Summary of quantitative evaluation of antimicrobial properties for control, Cu foil and NT-Cu samples. The reduction of the microbial population is expressed as log value at each point of time. The lack of antimicrobial action is denoted as “zero".

\begin{tabular}{|c|c|c|c|c|c|c|c|c|c|c|}
\hline \multirow[t]{3}{*}{ Organism } & \multirow[t]{3}{*}{ Sample } & \multicolumn{8}{|l|}{ Time [h] } & \multirow[t]{3}{*}{$\% R$} \\
\hline & & \multicolumn{2}{|l|}{$\mathbf{0}$} & \multicolumn{2}{|l|}{2} & \multicolumn{2}{|l|}{4} & \multicolumn{2}{|l|}{6} & \\
\hline & & $\log \mathrm{CFU} / \mathrm{mL}$ & Log red & $\log \mathrm{CFU} / \mathrm{mL}$ & Log red & $\log$ CFU/mL & Log red & $\log \mathrm{CFU} / \mathrm{mL}$ & Log red & \\
\hline \multirow[t]{3}{*}{ E. coli } & Control & 5.54 & 0.00 & 5.61 & 0 & 5.86 & 0 & 6.55 & 0 & 0 \\
\hline & Cu-NT & 5.57 & 0.00 & 5.50 & 0.07 & 4.77 & 0.80 & 3.90 & 1.67 & 97.8 \\
\hline & Cu-foil & 5.56 & 0.00 & 5.55 & 0.00 & 5.42 & 0.13 & 5.60 & 0 & 0 \\
\hline \multirow[t]{3}{*}{ S. aureus } & Control & 4.52 & 0.00 & 4.60 & 0 & 4.67 & 0 & 4.91 & 0 & 0 \\
\hline & $C u-N T$ & 4.43 & 0.00 & 4.18 & 0.26 & 2.60 & 1.83 & 0.00 & 4.43 & 100 \\
\hline & Cu-foil & 4.45 & 0.00 & 4.40 & 0.05 & 4.48 & 0 & 4.53 & 0 & 0 \\
\hline \multirow[t]{3}{*}{ S. sonnei } & Control & 5.20 & 0.00 & 5.30 & 0 & 5.78 & 0 & 6.34 & 0 & 0 \\
\hline & $\mathrm{Cu}-\mathrm{NT}$ & 5.03 & 0.00 & 5.01 & 0.01 & 4.11 & 0.91 & 3.79 & 1.24 & 94.2 \\
\hline & Cu-foil & 5.12 & 0.00 & 5.28 & 0 & 5.49 & 0 & 5.69 & 0 & 0 \\
\hline \multirow[t]{3}{*}{ S. enterica } & Control & 4.79 & 0.00 & 5.03 & 0 & 5.09 & 0 & 5.91 & 0 & 0 \\
\hline & Cu-NT & 4.96 & 0.00 & 5.10 & 0 & 4.62 & 0.34 & 3.97 & 0.99 & 89.9 \\
\hline & Cu-foil & 4.76 & 0.00 & 4.94 & 0 & 5.38 & 0 & 5.64 & 0 & 0 \\
\hline \multirow[t]{3}{*}{ C. albicans } & Control & 4.86 & 0.00 & 4.91 & 0 & 4.99 & 0 & 5.26 & 0 & 0 \\
\hline & $C u-N T$ & 4.61 & 0.00 & 4.60 & 0.01 & 3.64 & 0.97 & 3.60 & 1.01 & 90.3 \\
\hline & Cu-foil & 4.79 & 0.00 & 4.79 & 0.00 & 4.72 & 0.06 & 4.74 & 0.04 & 19.1 \\
\hline
\end{tabular}



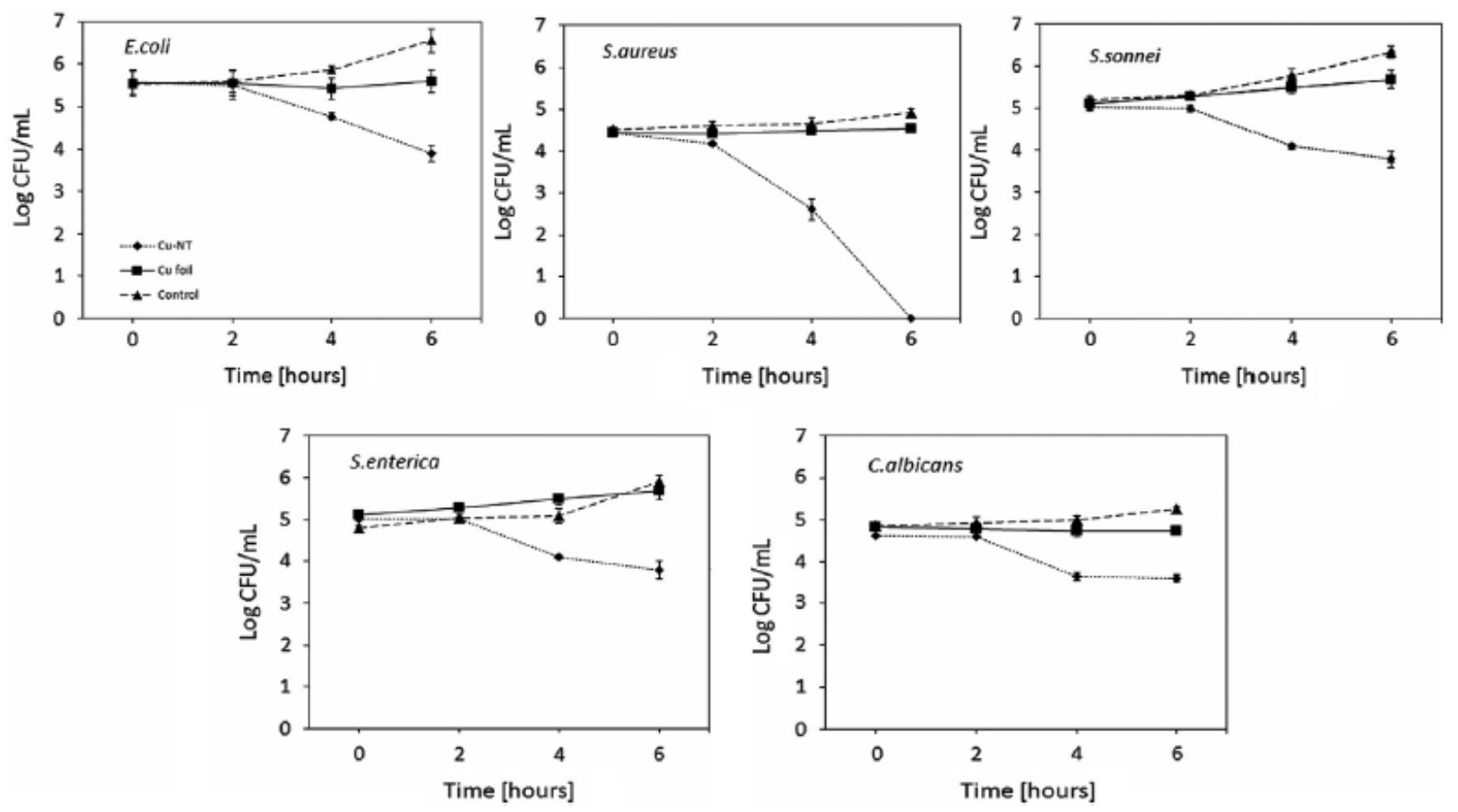

Fig. 2 Disc diffusion test results. A-E the zones of inhibition visible around the NT-Cu samples. F-J SEM micrographs of the samples’ surface.

NT-Cu showed good antimicrobial activity against all tested pathogens. However, the strength of this action depends on the type of the microorganism and their exposure time to NT-Cu. The most effective performance was observed against S.aureus, where antimicrobial action was observed after 2 hours of experiment (log red $=0.26$ ), increasing to 1.83 after 4 hours. After 6 hours over 99.99\% (log red $=4.43$ ) of the S.aureus population was inactivated. NT-Cu was less effective against Gram-negative organisms and yeast. After 2 hours of exposure minor antimicrobial action was observed against E.coli (log red 0.07), S.sonnei and C.albicans (log red 0.01), with S.enterica showing no sensitivity to NT-Cu's. The antimicrobial performance of the 
Cu NTs increased significantly after 4 hours of experiment and the final log reduction observed for E.coli, S.sonnei, S.enterica and C.albicans was 1.67; 1.24; 0.99; 1.01 respectively, corresponding to a reduction in the microbial population by $97.8 \%$ (E.coli) $94.2 \%$ (S.sonniei), 89.9\% (S.enterica) and 90.3\% (C.albicans). The reference Cu foil did not exhibit any significant antimicrobial action in suspension in all stages of experiment.

It is known that metal ions such as $\mathrm{Cu}^{+}$and $\mathrm{Ag}^{+}$can bind with cell walls and cause damage to the cellular functions by disrupting the osmotic pressure equilibrium. Furthermore, it has been shown that nanostructures of these materials are able to release ions that can cause local $\mathrm{pH}$ and conductivity changes. Compared to macro/micro structures, metal nanostructures, such as nanoparticles and nanowires/ nanotubes can bind easily with intracellular electron donating species such as cellular thiols, which are commonly found in enzymes [7, 8]. The Cu ions are small enough to disrupt bacterial cell membranes and gain entry in order to disrupt enzyme function. Similarly, secondary effects through changes in the surrounding charge environment by changing the local $\mathrm{pH}$ and conductivity, may also impact on the effectiveness of nanostructured metals against microorganisms [9]. In case of NT-Cu, the polycrystalline nature of the individual tube resulted in multifaceted crystal orientation exposed to the microorganisms, allowed them to bind. This can eventually affect the biological functions of bacteria within the bacterial cell. We believe that the presence of the holes in the structure (Fig. 1(b)) has no significant effect on activity when compared with the effect of nanostructuring. When the metabolic activity is disrupted the cell can no longer maintain osmotic pressure and cellular substances can leak out of the cellular matrix causing necrosis. In addition it is reported that $\mathrm{Cu}$ ions induce the creation of reactive oxygen species (ROS), which cause further cell damage [10]. Creation of reactive 
oxygen species is also reported [10] to be mediated as a result of the redox cycling between the various copper species such as $\mathrm{Cu}(0), \mathrm{Cu}(\mathrm{I})$, and $\mathrm{Cu}(\mathrm{II})$.

\section{Conclusion}

Vertically aligned $\mathrm{Cu}$ nano-tubular arrays were successfully obtained via template based electrodeposition. They show strong anti-microbial activity towards S.aureus after 4 hours, with other microbial populations requiring a longer exposure of 6 hours. The results show that novel NT-Cu structures have good potential for applications as antimicrobial materials or as specific microbial sensors. Further work will allow optimising the anti-microbial activity of these structures.

\section{References}

[1] Li Q, Mahendra S, Lyon DY, Brunet L, Liga MV, Li D, et al. Antimicrobial nanomaterials for water disinfection and microbial control: Potential applications and implications. Water Research. 2008;42:4591-602.

[2] Rengifo-Herrera JA, Pulgarin C. Photocatalytic activity of N, S co-doped and N-doped commercial anatase TiO2 powders towards phenol oxidation and E. coli inactivation under simulated solar light irradiation. Solar Energy. 2010;84:37-43.

[3] Ren G, Hu D, Cheng EWC, Vargas-Reus MA, Reip P, Allaker RP. Characterisation of copper oxide nanoparticles for antimicrobial applications. International Journal of Antimicrobial Agents. 2009;33:587-90.

[4] Jose Ruben M, Jose Luis E, Alejandra C, Katherine H, Juan BK, Jose Tapia R, et al. The bactericidal effect of silver nanoparticles. Nanotechnology. 2005;16:2346. 
[5] Khan SA, Singh AK, Fan Z, Senapati D, Ray PC. Designing distance dependent SERS assay for monitoring photothermal antibacterial activity response. Chemical Communications. 2012;48:11091-3.

[6] Pang H, Gao F, Lu Q. Morphology effect on antibacterial activity of cuprous oxide. Chemical Communications. 2009;0:1076-8.

[7] Smetana AB, Klabunde KJ, Marchin GR, Sorensen CM. Biocidal Activity of Nanocrystalline Silver Powders and Particles. Langmuir. 2008;24:7457-64.

[8] Feng QL, Wu J, Chen GQ, Cui FZ, Kim TN, Kim JO. A mechanistic study of the antibacterial effect of silver ions on Escherichia coli and Staphylococcus aureus. Journal of Biomedical Materials Research. 2000;52:662-8.

[9] Sondi I, Salopek-Sondi B. Silver nanoparticles as antimicrobial agent: a case study on E. coli as a model for Gram-negative bacteria. Journal of Colloid and Interface Science. 2004;275:17782.

[10] Grass G, Rensing C, Solioz M. Metallic Copper as an Antimicrobial Surface. Applied and Environmental Microbiology. 2011;77:1541-7.

\section{Figures and Table Legends}

Fig. 1SEM images of (a) vertically aligned NT-Cu after removal of the template, inset TEM image of NT-Cu dispersed; (b) HRTEM image of single NT-Cu cut at an oblique angle to the long axis of the tube showing hole at B. (c) Defocus images taken from the boxed region in (b) showing Fresnel contrast from the grain boundary marked at D; (d) XRD pattern of the as deposited NT-Cu arrays. 
Fig. 2 Disc diffusion test results. A-E the zones of inhibition visible around the NT-Cu samples. F-J SEM micrographs of the samples’ surface.

Fig. 3 Quantitative evaluation of antimicrobial properties of NT-Cu.

Table 1. Summary of quantitative evaluation of antimicrobial properties for control, Cu foil and NT-Cu samples. The reduction of the microbial population is expressed as log value at each point of time. The lack of antimicrobial action is denoted as “zero".

Cite this as Razeeb et al, Materials Letters, 128, 2014, 60-63 